\title{
Regenerated rat skeletal muscle after periodic contusions
}

V.B. Minamoto,

S.R. Bunho and T.F. Salvini

\author{
Laboratório de Neurociências, U nidade de Plasticidade Muscular, \\ Departamento de Fisioterapia, U niversidade Federal de São Carlos, \\ São Carlos, SP, Brasil
}

\begin{abstract}
Correspondence
T.F. Salvini

Departamento de Fisioterapia

Universidade Federal de São Carlos

13565-905 São Carlos, SP

Brasil

Fax: +55-16-261-2081

E-mail: tania@ power.ufscar.br

Presented at the XVI Annual Meeting of the Federação de Sociedades de Biologia Experimental, Caxambu, MG, Brazil, August 29 - September 1, 2001.

Research supported by FAPESP

(No. 96/10112-3) and CNPq.
\end{abstract}

Received April 11, 2001

Accepted August 27, 2001

\section{Abstract}

In the present study we evaluated the morphological aspect and changes in the area and incidence of muscle fiber types of long-term regenerated rat tibialis anterior (TA) muscle previously submitted to periodic contusions. Animals received eight consecutive traumas: one trauma per week, for eight weeks, and were evaluated one $(\mathrm{N}=8)$ and four $(\mathrm{N}=9)$ months after the last contusion. Serial cross-sections were evaluated by toluidine blue staining, acid phosphatase and myosin ATPase reactions. The weight of injured muscles was decreased compared to the contralateral intact one (one month: $0.77 \pm 0.15 \mathrm{vs}$ $0.91 \pm 0.09 \mathrm{~g}, \mathrm{P}=0.03$; four months: $0.79 \pm 0.14$ vs $1.02 \pm 0.07 \mathrm{~g}$, $\mathrm{P}=0.0007$, respectively) and showed abundant presence of split fibers and fibers with centralized nuclei, mainly in the deep portion. Damaged muscles presented a higher incidence of undifferentiated fibers when compared to the intact one (one month: $3.4 \pm 2.1$ vs $0.5 \pm 0.3 \%$, $\mathrm{P}=0.006$; four months: $2.3 \pm 1.6$ vs $0.3 \pm 0.3 \%, \mathrm{P}=0.007$, respectively). Injured TA evaluated one month later showed a decreased area of muscle fibers when compared to the intact one $(\mathrm{P}=0.003)$. Thus, we conclude that: a) muscle fibers were damaged mainly in the deep portion, probably because they were compressed against the tibia; b) periodic contusions in the TA muscle did not change the percentage of type I and II muscle fibers; c) periodically injured TA muscles took four months to reach a muscle fiber area similar to that of the intact muscle.

Skeletal muscle contusion is common during sport activities and repetitive traumas are also frequent in athletes. Although the muscle is able to regenerate, recurrent muscle injuries could affect muscle performance.

Skeletal muscle regeneration has been well documented (1) and several studies have shown that skeletal muscles regenerate after contusion (2-5).

Long-term regeneration of skeletal muscle has also been studied using different meth- ods to induce muscle damage, such as local injection of hot Ringer solution (6) and intramuscular injection of Agkistrodon contortrix laticinctus (ACL) myotoxin (7). Both reports revealed that several weeks after injury the regenerated muscles showed an abundant presence of split fibers and fibers with centralized nuclei.

Previous reports have described differences in the temporal response of the regeneration process when the muscle was sub-
Key words

- Skeletal muscle

- Periodic contusion

- Rat

................... 
mitted to single or periodic traumas $(4,5)$. Although several aspects associated with muscle injury and muscle regeneration induced by trauma have been studied, longterm regeneration after periodic muscle contusions has not been evaluated in detail.

The purpose of the present study was to analyze the long-term regeneration process of the rat tibialis anterior (TA) muscle submitted to periodic contusions and evaluated at different times (one and four months) after the last contusion.

Seventeen male rats (Wistar) weighing $315 \pm 37 \mathrm{~g}$ were used. All animals had the right TA muscles traumatized once a week for eight weeks, and were evaluated one $(\mathrm{N}=$ $8)$ and four months $(\mathrm{N}=9)$ after the last trauma. Before each contusion, the animals were anesthetized by ether inhalation. Animals were held in the lateral position and the right hind limb was immobilized by extending the knee and by maximal foot plantar flexing, which allows the maximal stretch of TA muscle. Injury was produced by a metal mass falling through a metal guide tube, as previously described (5). The mass (200 g) was dropped from a height of $37 \mathrm{~cm}$ in the middle belly of TA muscles. Left muscles were not injured and were used as control.

Animals were weighed immediately before receiving the first contusion and on the day of sacrifice. Under deep anesthesia, both right and left TA muscles of both groups were removed and weighed. Absolute muscle weight and relative muscle weight, calculated as a percentage of the body weight, were determined.

Right and left TA muscles were immediately frozen in melting isopentane and stored in a freezer at $-56^{\circ} \mathrm{C}$. Frozen TA muscles were cut from a proximal to a distal region with a cryostat microtome (Microm HE 505) and serial cross-sections $(10 \mu \mathrm{m})$ were taken from the middle belly.

Alternate serial cross-sections were stained with $1 \%$ toluidine blue/ $1 \%$ borax or submitted to the following reactions: acid phos- phatase (8), myosin ATPase activity after alkali, pH 10.3 (9), or acid preincubation, pH 4.3 (10).

The area and the incidence of muscle fiber types were evaluated only in the deep region of TA muscles because this region was predominantly injured, with abundant presence of regenerated muscle fibers (Figure 1B) and undifferentiated fibers. On the other hand, the superficial region of TA muscles showed the rare presence of regenerated fibers (Figure 1A) when compared to the deep region. Also, some animals did not show signs of previous muscle injury in the muscle fibers located in the superficial area of the muscle.

Histological cross-sections were evaluated by light microscopy (Axiolab, Carl Zeiss, Oberkochen, Germany) to identify damaged and regenerated muscle fibers. Damaged muscle fibers were characterized by the presence of muscle fiber necrosis and areas with intensive cellular infiltration. Peripheral or generalized basophilic areas were also common and reflected ribosome proliferation involved in protein synthesis. We also observed some fibers with centralized nuclei and prominent nucleoli, also related to protein synthesis. Another aspect of damaged muscle fibers is the activity of acid phosphatase, which is the main histochemical criterion to identify muscle fiber necrosis. Regenerated muscle fibers characterized by the presence of split fibers and centralized nuclei predominated in the traumatized muscles (Figure 1). Split fibers are described as fibers with one or more fragments. Normal muscle fibers have peripheral nuclei, but the regenerated regions of the fibers usually show centralized nuclei (5).

Undifferentiated muscle fibers are classified as fibers that express different types of myosin heavy chain and are usually rare, but not absent, in normal muscle and their presence suggests a change from one muscle fiber type to another. There is an increase in the incidence of undifferentiated fibers dur- 
ing muscle regeneration (11).

The incidence of muscle fiber types was identified using images from a video printer (Color Video Printer Mavigraph, Sony, Tokyo, Japan) linked to a light microscope. A total of $2221 \pm 566$ fibers from the deep region of each TA muscle were analyzed using histological cross-sections submitted to myosin ATPase reactions, in which muscle fibers were identified as type I, II or undifferentiated. The area of the same muscle fibers was evaluated using a computer software (Vinspec).

The paired and unpaired Student $t$-test (significant when $\mathrm{P} \leq 0.05$ ) was used for statistical analysis.

The results of this study showed that despite the periodic contusions there was body weight gain (one month: $140 \pm 27$ g, four months: $221 \pm 54 \mathrm{~g}$; Table 1), indicating that periodic contusions in TA muscle did not impair the body weight gain. Possible changes in the feeding behavior and in the growth of the animals may also have occurred, but these aspects were not evaluated in this study.

With respect to muscle weight, the intact TA muscles of both groups submitted to periodic muscle contusions were heavier than the injured ones when both the absolute (one month: $0.91 \pm 0.09$ vs $0.77 \pm 0.15 \mathrm{~g}, \mathrm{P}=0.03$; four months: $1.02 \pm 0.07$ vs $0.79 \pm 0.14 \mathrm{~g}$, $\mathrm{P}=0.0007$, respectively) and relative (one month: $0.187 \pm 0.01$ vs $0.159 \pm 0.03 \%, \mathrm{P}=$ 0.02 ; four months: $0.199 \pm 0.01$ vs $0.156 \pm$ $0.03 \%, \mathrm{P}=0.0008$, respectively) muscle weights were evaluated (Table 1).

This difference in muscle weight between injured TA and intact TA muscles indicates that periodic contusions decreased muscle weight in long-term regenerated muscles.

Another factor that could be related to the muscle weight differences is the possible hypertrophy induced by the overload in the contralateral hind limb, probably required to compensate for the hypoactivity of the injured hind limb. It is well known that over- use can cause muscle hypertrophy (for a review, see 12).

An interesting result was the absence of significant differences between the weight of injured TA muscles evaluated one and four months after contusions, then showing that within a period of three months the traumatized muscles were not able to increase in weight, as observed in the intact contralateral muscles. Contralateral muscles were heavier in animals of the four-month
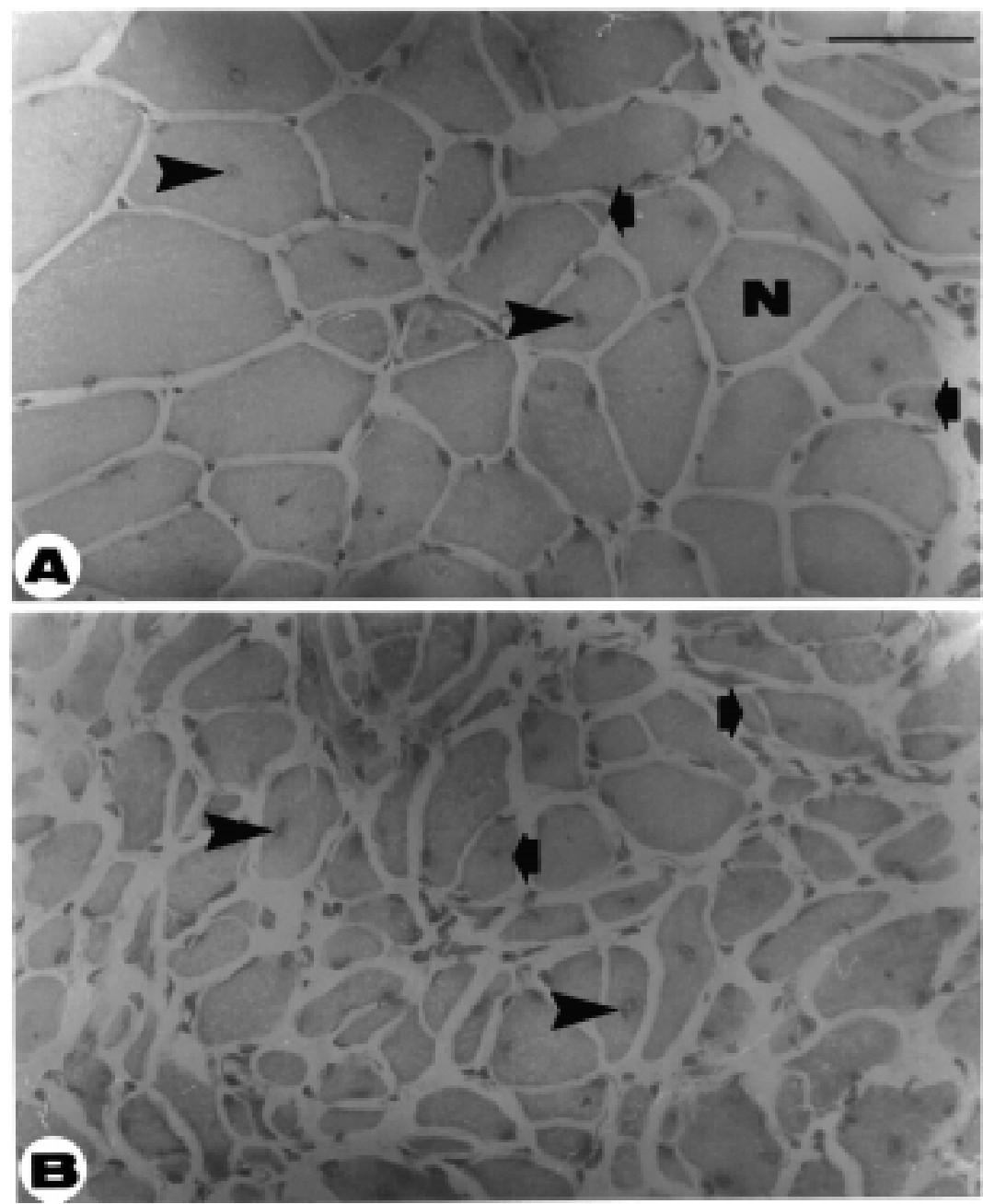

Figure 1. Histological cross-sections of the superficial (A) and deep (B) regions of tibialis anterior muscle, four months after periodic muscle injuries. Signs of regenerated muscle fibers are identified by the presence of centralized nuclei (arrowheads) and split fibers (arrows). Note large spaces among the muscle fibers in the deep region of the muscle (B). Also, the signs of previous muscle injury are more abundant in the deep region of the muscle (B) when compared to the superficial region (A). $N=$ normal fibers. Toluidine blue stain. Bar: $42 \mu \mathrm{m}$. 
group, when compared to the one-month group (Table 1). These results show that periodic injuries inhibited the gain of muscle weight.

Previous studies have shown different results related to muscle weight. In our laboratory, soleus muscle submitted to periodic contusions showed an increase in muscle weight when evaluated six days after periodic contusions, but no difference after 30 days (5). The authors also observed an increase in the injured muscle weight after periodic damage and suggested that this was a consequence of connective tissue proliferation (13). When the weight of the gastrocnemius muscle was determined after contusion an increase in weight was observed, due to the accumulation of edema and hemorrhage (3). On the other hand, in another study, no increase in the weight of injured muscles was observed and it was suggested that protein depletion equaled the increased weight due to edema and hemorrhage (2).

Table 1. Body and tibialis anterior (TA) muscle weight of the one- and four-month groups of animals.

\begin{tabular}{lll}
\hline & One month & Four months \\
\hline $\begin{array}{l}\text { Body weight }(g) \\
\text { Initial }\end{array}$ & $344 \pm 29^{*}$ & $289 \pm 20$ \\
$\quad$ Final & $484 \pm 44$ & $510 \pm 36$ \\
$\quad$ Total gain & $140 \pm 27$ & $221 \pm 54$ \\
$\begin{array}{l}\text { Muscle weight (g) } \\
\text { Injured TA }\end{array}$ & \\
$\quad$ Intact TA & $0.77 \pm 0.15^{* *}$ & $0.79 \pm 0.14^{* * *}$ \\
Relative muscle weight (\%) & $0.91 \pm 0.09^{\#}$ & $1.02 \pm 0.07$ \\
$\quad$ Injured TA & & \\
Intact TA & $0.159 \pm 0.03^{+}$ & $0.156 \pm 0.03^{++}$ \\
\hline
\end{tabular}

Results are reported as means $\pm \mathrm{SD}$.

$* \mathrm{P}=0.0003$ compared to the four-month group (unpaired Student $\mathrm{t}$-test).

$* * \mathrm{P}=0.03$ compared to the intact TA of the one-month group (paired Student t-test). $* * * \mathrm{P}=0.0007$ compared to the intact TA of the four-month group (paired Student $\mathrm{t}$ test).

$+P=0.024$ compared to the intact TA of the one-month group (paired Student t-test). $+P=0.0008$ compared to the intact TA of the four-month group (paired Student $t$ test).

\#P $=0.01$ compared to the intact TA of the four-month group (unpaired Student t-test). \# $=0.04$ compared to the intact TA of the four-month group (unpaired Student $\mathrm{t}$ test).
Some factors may explain these differences in the literature, such as the muscle characteristic (slow or fast muscles), the protocols used to produce contusions and the period of muscle evaluation.

A previous study has reported that during the first days after periodic contusions the injured muscle showed bundles of degenerated fibers with necrosis, cellular infiltration, basophilic fibers and acid phosphatase activity (5). However, in the present study there was a predominance of regenerated muscle fibers with split fibers and centralized nuclei when muscle regeneration was evaluated one and four months later.

In both groups evaluated (one and four months), injured TA muscles showed the predominant presence of split fibers and fibers with centralized nuclei, mainly in the deep portion of the muscles (Figure 1B). This portion was the most damaged probably due to the model of induced contusion, in which at the time of impact the soft tissues suffer a pressure (3). This stress should be more evident in the deeper fibers, as they absorb the pressure and are compressed against the tibia.

Fibers with signs of regeneration were also found in some bundles of the superficial portions of TA muscles, usually mixed with normal fibers (Figure 1A). It was interesting to observe that some traumatized muscles showed signs of degenerated muscle cells, mainly cellular infiltration, basophilia and acid phosphatase activity, even one and four months after the last contusion. This study differs from a previous report in which degenerated muscle fibers were observed only during the first week after contusion (14). Our results were probably caused by the repetitive muscle fiber lesions, since previous work showed a normal morphological pattern of muscle fibers 21 days after a single contusion (2).

Although all injured TA muscles of the one-month group showed a high incidence of muscle injury, injured TA of the four- 
month group did not show a similar incidence of muscle damage among the animals, i.e., four muscles showed a large incidence of signs of damage, three showed little damage, and two showed a normal muscular pattern. The similar morphological pattern of the injured TA muscle of the one-month group suggests that these animals were in a similar stage of muscle regeneration. The absence of previous signs of injury in some muscles of the four-month group may have been due to the fact that this period was enough to promote complete muscle regeneration. Also, animals of the four-month group were younger than animals of the onemonth group when the experiment started and some reports have shown that animal age influences the regeneration process, i.e., injured muscles regenerate better in young than in old animals (15). Individual differences among regenerated skeletal muscles after trauma have also been described (5).

The injured TA muscles of both groups showed a significant increase in the number of undifferentiated muscle fibers when compared to the intact one (one month: $3.41 \pm$ 2.12 vs $0.51 \pm 0.27 \%, \mathrm{P}=0.006$; four months: $2.27 \pm 1.65$ vs $0.27 \pm 0.3 \%, \mathrm{P}=0.007$, respectively). There were no significant differences when type I and II muscle fibers were compared to contralateral intact muscles (Table 2).

Previous reports have shown an increased number of undifferentiated fibers and a decreased number of type II muscle fibers on the soleus of mice after running-induced muscle damage. This change was also associated with an increased number of axonal sprouts and indicates axonal remodelation (16). Similar results were observed in the deep portion of the gastrocnemius muscle (fast-twitch) of mice 21 days (17) and 8 months (7) after muscle damage induced by ACL myotoxin.

In the present study, we did not observe changes in the number of muscle fibers from type II to type I, probably because of the predominance of type II fibers in the TA muscle, which is composed of $95 \%$ type II muscle fibers (18).

Animals of the one-month group showed a significantly smaller area of type I and II muscle fibers in injured TA when compared to the intact TA (type I: $1883 \pm 391$ vs 3021 $\pm 484 \mu \mathrm{m}^{2}$; type II: $2415 \pm 1108$ vs $4405 \pm$ $817 \mu \mathrm{m}^{2}, \mathrm{P}=0.003$, respectively), a result probably related to the abundant presence of split fibers in the regenerated muscles. Also, possible hypertrophy associated with the overuse of the contralateral intact muscles should be considered, caused by probable disuse periods in the injured hind limbs after trauma. No changes were found in the area of undifferentiated muscle fibers in the onemonth group.

On the other hand, injured muscles of the four-month group showed a significant increase only in the area of undifferentiated fibers when compared to intact muscle (4094 \pm 1291 vs $2686 \pm 696 \mu \mathrm{m}^{2}, \mathrm{P}=0.019$, respectively). This is an interesting result because, although immature, these fibers

Table 2. Type I, II and undifferentiated (Undif.) muscle fibers and area of muscle fibers of the one- and four-month groups of animals.

\begin{tabular}{|c|c|c|c|c|}
\hline & \multicolumn{2}{|c|}{ One month } & \multicolumn{2}{|c|}{ Four months } \\
\hline & Injured TA & Intact TA & Injured TA & Intact TA \\
\hline \multicolumn{5}{|c|}{ Muscle fiber types (\%) } \\
\hline I & $6.9 \pm 3.7$ & $7.4 \pm 1.3$ & $6.2 \pm 3.0$ & $5.3 \pm 2.3$ \\
\hline II & $89.6 \pm 5.1$ & $92.0 \pm 1.5$ & $91.5 \pm 4.3$ & $94.3 \pm 2.3$ \\
\hline Undif. & $3.4 \pm 2.1^{*}$ & $0.5 \pm 0.3$ & $2.3 \pm 0.06^{* *}$ & $0.3 \pm 0.3$ \\
\hline \multicolumn{5}{|c|}{ Muscle fiber area $\left(\mu \mathrm{m}^{2}\right)$} \\
\hline I & $1883 \pm 391^{+\#}$ & $3021 \pm 484$ & $4039 \pm 908$ & $3653 \pm 856$ \\
\hline II & $2415 \pm 1108^{+\#}$ & $4405 \pm 817^{\# \#}$ & $5977 \pm 1025$ & $5807 \pm 1102$ \\
\hline Undif. & $1829 \pm 362^{\#}$ & $2337 \pm 555$ & $4094 \pm 1291^{++}$ & $2686 \pm 696$ \\
\hline
\end{tabular}

Results are reported as means $\pm \mathrm{SD}$. TA = tibialis anterior muscle. $* \mathrm{P}=0.006$ compared to the intact TA of the one-month group (paired Student t-test). $* * \mathrm{P}=0.007$ compared to the intact TA of the four-month group (paired Student t-test). $+P=0.003$ compared to the intact TA of the one-month group (paired Student t-test). $+P=0.019$ compared to the intact TA of the four-month group (paired Student t-test). \#P $\leq 0.00002$ compared to the injured TA of the four-month group (unpaired Student $\mathrm{t}$ test).

\# 0.01 compared to the intact TA of the four-month group (unpaired Student ttest). 
reach normal size. No differences were identified in the area of types I and II between injured and intact muscles (Table 2).

The results obtained for the four-month group suggest that a period of four months was enough for the injured muscle fibers to reach an area similar to that of the intact muscles, with injured TA of the four-month group showing a significantly larger area when compared with the one-month group. This is probably a consequence of intense protein synthesis in the muscle fibers during the four months after the traumas.

An interesting result was that, although there was an increase in the area of muscle fibers of the injured muscles, this increase was not followed by an increase in total muscle weight. Recent reports evaluated the injured muscle some weeks later and found a partial or total destruction of a small subpopulation of muscle fibers, which reflects a muscle volume loss (19). Then, we may suggest that muscle weight did not match the muscle fiber area because the destruction of some muscle fibers affected total muscle weight, although the preserved muscle fibers showed hypertrophy.

\section{References}

1. Bodine-Fowler S (1994). Skeletal muscle regeneration after injury: an overview. J ournal of Voice, 8: 53-62.

2. Fisher BD, Baracos VE, Shnitka TK, Mendryk SW \& Reid DC (1990). Ultrastructural events following acute muscle trauma. Medicine and Science in Sports and Exercise, 22: 185-193.

3. Crisco J J , J okl P, Heinen GT, Connell MD \& Panjabi MM (1994). A muscle contusion injury model. American J ournal of Sports Medicine, 22: 702-710.

4. Louboutin JP, Fichter-Gagnepain V, Pastoret C, Thaon E, Noireaud J , Sébille A \& Fardeau M (1995). Morphological and functional study of extensor digitorum longus muscle regeneration after iterative crush lesions in MDX mouse. Neuromuscular Disorders, 5: 489-500.

5. Minamoto VB, Grazziano CR \& Salvini TF (1999). Effect of single and periodic contusion on the rat soleus muscle at different stages of regeneration. Anatomical Record, 254: 281-287.

6. Schmalbruch H (1976). Muscle fibre splitting and regeneration in diseased human muscle. Neuropathology and Applied Neurobiology, 2: 3-19.

7. Salvini TF, Morini $\mathrm{CC}$, Selistre de Araújo HS \& Ownby CH (1999). Long-term regeneration of fast and slow murine skeletal muscles after induced injury by $\mathrm{ACL}$ myotoxin isolated from Agkistrodon contortrix laticinctus (broad-banded copperhead) venom. Anatomical Record, 254: 521-533.

8. Barka T \& Anderson PJ J (1962). Histochemical methods for acid phosphatase using hexazonium pararosaniline as coupler. J ournal of Histochemistry and Cytochemistry, 10: 741-753.

9. Butler J \& Cosmos E (1981). Enzymic markers to identify muscle-nerve formation during embryogenesis: modified myosin ATPase and silver-cholinesterase histochemical reactions. Experimental Neurology, 73: 813-816.

10. Brooke MH \& Kaiser KK (1970). Muscle fiber types: How many and what kind? Archives of Neurology, 23: 369-397.

11. Pette D \& Staron RS (1997). Mammalian skeletal muscle fiber type transitions. Intemational Review of Cytology, 170: 143223.

12. Lieber RL (1992). Skeletal Muscle Structure and Function. Implications for Rehabilitation and Sports Medicine. Williams \& Wilkins, Baltimore, MD, USA.

13. Tamaki T, Akatsuka A, Uchiyama S, Uchiyama Y \& Shiraishi T (1997). Appearance of complex branched muscle fibers is associated with a shift to slow muscle characteristics. Acta Anatomica, 159: 108-113.

14. Hurme T\& Kalimo H (1992). Activation of myogenic precursor cells after muscle injury. Medicine and Science in Sports and Exercise, 24: 197-205.

15. Devor ST \& Faulkner J A (1999). Regeneration of new fibers in muscles of old rats reduces contraction-induced injury. J oumal of Applied Physiology, 87: 750756.

16. Wernig A, Salvini TF \& Irintchev A (1991). Axonal sprouting and changes in fibre types after running-induced muscle damage. J ournal of Neurocytology, 20: 903913.

17. Morini CC, Pereira ECL, Selistre de Araújo HS, Ownby CH \& Salvini TF (1998). Injury and recovery of fast and slow skeletal muscle fibers affected by ACL myotoxin isolated from Agkistrodon contortrix laticinctus (broad-banded copperhead) venom. Toxicon, 36: 1007-1024.

18. Delp MD \& Duan C (1996). Composition and size of type I, IIA, IID/X, and IIB fibers and citrate synthase activity of rat muscle. J ournal of Applied Physiology, 80: 261270.

19. Foley JM, J ayaraman RC, Prior BM, Pivarnik J M \& Meyer RA (1999). MR measurements of muscle damage and adaptation after eccentric exercise. J ournal of Applied Physiology, 87: 2311-2318. 